\title{
SATISFACCIÓN LABORAL DEL PROFESORADO NO UNIVERSITARIO SEGÚN TITULARIDAD DEL CENTRO
}

\author{
JOB SATISFACTION OF TEACHERS ACCORDING \\ TO THE OWNERSHIP OF THE SCHOOL
}

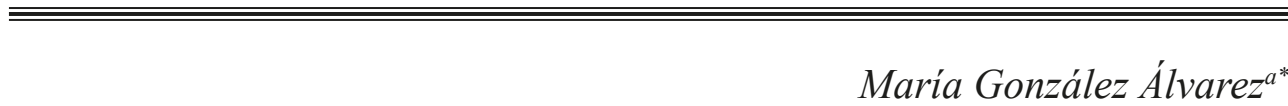

Fechas de recepción y aceptación: 9 de mayo de 2020 y 8 de noviembre de 2020

DOI: https://doi.org/10.46583/edetania_2020.58.635

Resumen: El presente trabajo tiene como objetivo realizar un estudio exploratorio sobre la satisfacción laboral del profesorado de enseñanzas no universitarias según la titularidad del centro, analizada respecto a cargos directivos, relaciones interpersonales, instalaciones, formación y situaciones de acoso, maltrato o agresión. Se hizo una consulta online mediante un protocolo elaborado ad hoc que fue cumplimentado por 2738 docentes. Se realizaron análisis descriptivos, diferencia de medias y análisis multivariado, así como un análisis factorial confirmatorio cuyos índices más significativos son: $\mathrm{PCMIN} / \mathrm{DF}=1.979$; $\mathrm{RMSEA}=.057$; $\mathrm{NFI}=.904 ; \mathrm{CFI}=.949 ; \mathrm{TLI}=.941$ y $\mathrm{PNFI}=.777$ y un coeficiente de Cronbach de .955 . Considerando que el modelo cuenta con buena consistencia interna e índices de bondad de ajuste adecuados, la satisfacción en general se considera media-alta. Los docentes de los centros privados se manifiestan más satisfechos que los públicos respecto a su formación, cargos directivos, función tutorial, consejo escolar y aspectos laborales. El profesorado sufre situaciones de acoso, maltrato o agresión. Se considera que debe profundizarse en el estudio del importante tema de la satisfacción laboral del profesorado, correspondiendo a las administraciones implementar programas de análisis y ayuda al respecto. Procede seguir investigando la satisfacción laboral en función de las diferencias en relación con la titularidad del centro.

Palabras clave: satisfacción laboral, profesor, enseñanza pública, enseñanza privada.

a Profesora de Educación Secundaria. IES La Quintana. Ciaño-Langreo (Asturias)

* Correspondencia: Instituto de Educación Secundaria La Quintana. Calle Jaime Alberti, s/n 33900 Langreo. Asturias. España.

E-mail: maria.gzaz@gmail.com 
Abstract: The goal of this paper is to conduct an exploratory study on the job satisfaction of teachers based on the ownership of the school and analyzed regarding positions, interpersonal relationships, facilities, training and situations of harassment, abuse or assault. An online survey was launched using an ad hoc protocol that was filled in by 2738 teachers. To this purpose, descriptive analysis, media difference, and multivariate analysis were carried out as well as a factorial confirmatory analysis whose most meaningful indexes are: PCMIN/ $\mathrm{DF}=1.979 ; \mathrm{RMSEA}=.057$; NFI=.904; $\mathrm{CFI}=.949 ; \mathrm{TLI}=.941 \mathrm{y} \mathrm{PNFI}=.777$ and a Conbrach's coefficient of .995 . The model counts on good internal consistency and an accurate goodness fit index. In general terms, the level of satisfaction is considered as medium-high. Teachers in private schools feel more satisfied about their training, their responsibility in management positions and as head teachers, their membership of the school board and about labor issues than teachers in public schools. Teachers often experience harassment, mistreatment or aggression, reaching a higher percentage in public schools. The important issue of the job satisfaction of teachers should be deeply studied, being the administrations responsible for implementing analysis and assistance programs. It is necessary to analyze the differences established in relation to the ownership of the school and the job satisfaction. Further research is needed according to these differences.

Keywords: job satisfaction, teacher, public education, private education.

\section{INTRODUCCIÓN}

La satisfacción laboral del profesorado se relaciona en diversos trabajos con la calidad de la enseñanza (Anaya-Nieto y López-Martín, 2014; Banerjee, Stearns, Moller y Mickelson, 2017; Demirtaú, 2010; Gil-Flores, 2017; Persevica, 2011). Dicha satisfacción se presenta hoy en la investigación educativa como un tema de gran interés (Cantón-Mayo y Téllez-Martínez, 2016; Güell, 2014).

En relación con la definición de la satisfacción laboral, Cantón-Mayo y Téllez-Martínez señalan que para hacer referencia a esta se utilizan una diversidad de términos, considerando que se "percibe como una actitud, una emoción, un sentimiento y relacionada con el clima, con la salud mental y el equilibrio personal" (2016, p. 215). Señalan Anaya-Nieto y López-Martín que la satisfacción laboral "se refiere a cómo la gente se siente en relación con su actividad laboral o con los diferentes aspectos de su trabajo" (2014, p. 436). Por otro lado, para dichos autores los factores relevantes que explican la satisfacción laboral varían de unas propuestas teóricas a otras, indican distin- 
tos modelos según la mayor atención a diferentes aspectos de dicha satisfacción, tales como las características del trabajo, la autoeficiencia, características personales, expectativas, metas perseguidas o al ajuste persona-ambiente. En el presente trabajo se pretende prestar atención a distintos aspectos de la satisfacción laboral del profesorado no universitario de España con referencia a aportaciones de los distintos modelos señalados y a la experiencia profesional propia. Se refieren a la satisfacción laboral del profesorado: en sus relaciones con las instituciones y la comunidad educativa, con las instalaciones y recursos del centro, la formación recibida, aspectos laborales así como el reconocimiento y valoración recibidos por parte de las instituciones y comunidad educativa.

En relación con el constructo sobre la satisfacción laboral señala Güell que "es pluridimensional siendo, por tanto, varias las dimensiones que contribuyen a conseguirla" (2015, p. 22). En los estudios longitudinales se constata que la satisfacción laboral del profesorado ha disminuido en los últimos años (Anaya-Nieto y López-Martín, 2014; Gil-Flores, 2017; Klassen \& Anderson, 2009; MetLife Foundation, 2012).

La satisfacción laboral del profesorado en función de la titularidad del centro es un ámbito escasamente analizado en la investigación educativa. Se entiende que su estudio puede proporcionar importante información en relación con el constructo de la satisfacción laboral. En España la red de centros educativos que imparten enseñanzas no universitarias se conforma con centros de titularidad pública y centros de titularidad privada. Se debate con frecuencia la necesidad y existencia de esta doble red, vinculando las divergencias fundamentalmente a planteamientos de tipo ideológico y a aspectos como la financiación de la red privada por parte de la administración. Se entiende por algunos que tan solo la red pública es la que debe estar sostenida con recursos públicos. Aunque con menor interés, se suscita en ocasiones el tema de la calidad de la enseñanza que proporcionan los centros según su titularidad.

El nivel de satisfacción laboral del profesorado se considera "alto" en distintos trabajos (Demirtaú, 2010; Naeem-Akhtara, Amir-Hashmib y HussainNaqvic, 2010; Peršēvica, 2011; Schwartz, 2017), siendo "medio-alto" para Anaya y Suárez (2007) y Larkin, Brantley-Dias y Lokey-Vega (2016); en otros estudios el nivel es considerado como "medio" (Muñoz-Menéndez, Gómez-Mármol y Sánchez-Alcaraz, 2017; Sotomayor-Atahuamán, 2020); y 
para Cantón-Mayo y Téllez-Martínez (2016) el nivel es “medio-bajo". En el informe Pisa (2019) se señala que, entre los 38 países participantes en el Talis 2018, España se encuentra entre los seis con profesorado más "altamente" satisfecho en su función docente.

La satisfacción laboral del profesorado en relación con la titularidad del centro está muy relacionada con el sistema educativo de cada país y la importancia y características que tengan en él los diferentes tipos de centros. Diversos trabajos indican que el profesorado de los privados se manifiesta más satisfecho que el de los públicos (Crossman y Harris, 2006; Gius, 2015; Naeem-Akhtara et al., 2010; Sungu, Ilgan, Parylo y Erdem (2014). En el trabajo de Nigama, Selvabaskar, Surulivel, Alamelu y Uthaya (2018) no se encuentran diferencias en función de la titularidad. En otros casos es el de los centros públicos el más satisfecho (Mitra, 2018). Para De Frutos, González, Maíllo, Peña y Riesco (2007) el profesorado de los centros privados en las escuelas católicas considera muy adecuada su satisfacción laboral.

La literatura ofrece amplia información en relación con las variables sociodemográficas del profesorado y su satisfacción laboral. Respecto al sexo no existe acuerdo. Para algunos autores las profesoras manifiestan una mayor satisfacción laboral que sus colegas varones (Anaya-Nieto y López-Martín, 2014; Gil-Flores, 2017; Gius, 2015; Martín-Ramos, 2015; Naeem-Akhtara et al., 2010; Raso-Sánchez, Sola-Martínez e Hinojo-Lucena, 2017; Xin y MacMillan, 1999); en otros trabajos no se encuentran diferencias (Crossman \& Harris, 2006; Muñoz-Menéndez et al., 2017; Skaalvik y Skaalvik, 2009). En algún caso son los profesores los que manifiestan mayor satisfacción laboral (Mitra, 2018). Convendría, en cualquier caso, tener en cuenta la situación general de la mujer y en particular en la profesión docente en los países y culturas de referencia de los distintos estudios.

Tampoco se encuentra unanimidad en las investigaciones realizadas respecto a la relación entre la satisfacción laboral y el tiempo de experiencia docente del profesorado. En distintos estudios se señala que con el aumento del tiempo de experiencia docente disminuye la satisfacción laboral del profesorado (Anaya-Nieto y López-Martín, 2014; Anaya-Nieto y Suárez, 2006; Gil-Flores, 2017; Skaalvic y Skaalvic, 2009; Xin y MacMillan, 1999); en otros trabajos no se encuentran diferencias (Crossman y Harris, 2006; Demirtaú, 2010). 
Respecto a la satisfacción laboral en función de las enseñanzas impartidas por el profesorado, no hay un total acuerdo en los trabajos realizados, pero se puede señalar que el mayor nivel de satisfacción laboral lo manifiesta el profesorado de educación infantil y el de educación primaria (Anaya-Nieto y López-Martín, 2014; Cantón-Mayo y Téllez-Martínez, 2016; Martín-Ramos 2015; Muñoz-Menéndez et al., 2017).

La satisfacción laboral en relación con los cargos de responsabilidad en el centro presenta diferentes resultados en los distintos trabajos. Mientras que para Papanastasiou y Zembylas (2005) el profesorado que ocupa puestos más altos en las instituciones de educación tiende a estar más satisfecho con su trabajo, para Ramery-Gelpi (2017) no hay diferencias significativas.

La satisfacción en las relaciones del profesorado con las instituciones y miembros de la comunidad educativa presenta distintas valoraciones. Se puede señalar que el profesorado se suele sentir poco satisfecho en sus relaciones con la administración educativa (Hernández-Silva, Pavez-Lizarraga, González-Donoso y Tecpan-Flores, 2017; Torres, 2010). Para Hernando-Mora y Sanz-Ponce (2015) los docentes reclaman más apoyo y recursos por parte de la administración y más colaboración de los servicios sociales. En distintos trabajos se indica que el profesorado se siente satisfecho en sus relaciones con el alumnado (Hernández-Silva et al., 2017; Torres, 2010; Veldman, Van Tartwijk, Brekelmas y Wubbles, 2013).

Respecto a la formación, diversos estudios estiman que el profesorado que participa en programas de formación continua se siente más satisfecho (Hernández-Silva et al., 2017; Martínez-Garrido, 2017). En este sentido, según RosGarrido y García-Rubio (2016), para garantizar la calidad educativa se debe proporcionar una adecuada formación inicial y permanente al profesorado.

En distintas investigaciones las condiciones del lugar de trabajo se relacionan con la satisfacción laboral, siendo esta mejor considerada cuanto mejor valorado es el centro (National Center For Education Statistics, 1997; Santos-Rego, Godás-Otero, Lorenzo-Moledo y Gómez-Fraguela, 2010). Por otro lado, según Anaya-Nieto y López-Martín (2014) el profesorado más veterano tiene mayor satisfacción en relación con las condiciones de vida asociadas a la facilidad de desplazamiento entre el domicilio y el centro. Respecto al salario percibido algunos trabajos señalan que tiene un efecto positivo sobre la satisfacción laboral (Franco-López, López-Arellano y Arango-Botero, 2020; 
Persevica, 2011), en otros casos el salario no se relaciona con la satisfacción profesional (Hanushek, Kain y Rivkin, 2004).

Aspectos de gran interés en la investigación educativa en la actualidad son la ansiedad, el estrés, la carga de trabajo y el burnout en relación con la satisfacción laboral. Para algunos autores, las condiciones de trabajo están relacionadas con el estrés y la ansiedad (Collie, Shapka y Perry, 2012; Ferguson, Frost y Hall, 2012), los cuales disminuyen la satisfacción laboral (Klassen y Chiu 2010; Liu y Ramsey, 2008). Para Hernando-Mora y Sanz-Ponce (2015) el $15 \%$ del profesorado de educación secundaria entiende que la violencia del aula le desmotiva, siendo mayor dicho porcentaje en el caso de los centros públicos que en el de los privados.

\section{Metodología}

\subsection{Muestra}

Se realizó una consulta al profesorado de educación no universitaria de las diferentes comunidades autónomas de España con el objetivo de conocer su satisfacción respecto a diversos aspectos relacionados con su actividad laboral y en función de la titularidad del centro. A los efectos se extrajo al azar una muestra de centros del Registro Estatal de Centros Docentes no Universitarios del Ministerio de Educación, en función de las enseñanzas impartidas, de su titularidad y de la población de las distintas provincias. El cuestionario puede ser cumplimentado en 15 minutos. Dicha muestra consta de 2425 centros inscritos en dicho Registro, siendo el 78,2 \% de titularidad pública y el 21,2 \% privados o privados-concertados, a los cuales se les envió un cuestionario, elaborado ad hoc, y una carta solicitando la colaboración en el trabajo, mediante correo electrónico utilizando la aplicación Mailchimp. Las respuestas se recogían en una aplicación de Google. El procedimiento utilizado para enviar el cuestionario presenta inconvenientes como la desconfianza que genera recibir un correo electrónico del que se desconoce su origen, siendo eliminado en muchas ocasiones sin haber procedido a su apertura, lo que reduce así el número de posibles respuestas. Supone, asimismo, que al realizar el envío al correo electrónico del centro es decisión del responsable de este reenviarlo o 
no al profesorado. Por otro lado, tiene como ventajas el poder enviar un elevado número de mensajes a centros elegidos con los procedimientos señalados y con la garantía de anonimato. Según la información que nos proporciona la aplicación utilizada para realizar el envío, este fue abierto en el 32,21\% de los casos. No se tienen evidencias, como es obvio, de cuántos fueron los mensajes leídos y cuántos fueron reenviados al profesorado.

Cumplimentaron el protocolo 2738 docentes de los cuales el 64,7\% son mujeres, el $34,3 \%$ varones y el $1,1 \%$ no responde. Su edad media es de 46.2 años, desviación típica 8,8 años, en adelante DT (profesoras 45,5 años y profesores 47,5 años, grado de significación .001, en adelante $\rho$ ). Su experiencia profesional media es de 17,7 años, DT=10,4 años (profesoras 17,1 y profesores 18.9, $\rho .001)$. El 91,7 ejerce en centros públicos, el 8,0 \% en privadosconcertados y el .3\% en privados. El 5,7\% imparte su docencia en educación infantil, el 20,5 \% en educación primaria, el 43,4 \% en educación secundaria obligatoria (en adelante ESO), el 15,1 \% en bachillerato y el 15,3 \% en formación profesional (en adelante FP). La media de alumnos y alumnas de los centros en los que llevan a cabo sus tareas profesionales es de 427 .

\subsection{Variables e instrumentos}

Para realizar el estudio se ha elaborado un protocolo específico que fue analizado en su primera fase mediante "el juicio de expertos", siendo estos dos inspectores de educación, tres directores y directoras de centros (dos de centros públicos y uno privado-concertado) y cinco profesores y profesoras (cuatro ejerciendo en centros públicos y uno en privado-concertado, siendo uno de cada enseñanza), los cuales valoraron los ítems del cuestionario inicial en una escala tipo Likert de 0 a 10 puntos, siendo el 0 el menor valor y el 10 el máximo. Se eliminan los ítems con valoración media inferior a siete puntos. El nuevo cuestionario volvió a ser analizado por los "expertos" prestando atención a la calidad de la redacción de los ítems y a aspectos no incluidos en los análisis estadísticos. Con las últimas aportaciones se elaboró el cuestionario definitivo que se conforma con 38 ítems, además de las variables sociodemográficas, la satisfacción en cargos de responsabilidad en el centro y situaciones de acoso, maltrato o agresión sufridas por el profesorado. El 
cuestionario definitivo quedó constituido por: 1) variables sociodemográficas; 2) satisfacción en cargos de responsabilidad; 3) satisfacción en las relaciones interpersonales; 4) satisfacción con las instalaciones y recursos del centro; organización y funcionamiento del centro, ambiente de trabajo y espacios disponibles; 5) satisfacción con la formación recibida; 6) satisfacción con aspectos laborales, posibilidades de participación, reconocimiento y valoración del trabajo del profesorado, posibilidades de promoción profesional, satisfacción laboral actual en relación con la de hace cinco años; 7) posibles situaciones de acoso, maltrato o agresión y grado sufridos.

La información se analiza mediante descriptivos, diferencia de medias y análisis multivariado, utilizando el método Scheffé y análisis factorial confirmatorio. El grupo de los centros privados-concertados y privados se incluyen ambos bajo la denominación de "privados-concertados". Las escalas del cuestionario tienen un recorrido de 0 a 10 puntos, siendo 0 el menor valor y 10 el máximo. La experiencia docente se distribuye en tres grupos semejantes (hasta 12 cursos, de 13 a 22 cursos y 23 o más cursos). El tamaño del centro se estudia en tres grupos, considerando el número de alumnos y alumnas (hasta 160, de 161 a 500 y 501 o más).

Se realiza un análisis factorial confirmatorio con los 38 ítems que componen el cuestionario y se eliminan cinco cuyas cargas factoriales son menores de .60, quedando un total de 33 ítems. Los eliminados se refieren a la satisfacción con "el profesorado del departamento o ciclo", "la formación inicial recibida en los estudios universitarios respecto al trabajo como profesor o profesora", "la ubicación del centro en el que se ejercen las funciones", "las retribuciones que se perciben como profesor o profesora" y "el tiempo que se tarda en llegar desde el domicilio al centro de trabajo".

En relación con la validez del constructo de los 33 ítems, la medida de adecuación muestral de Kaiser-Meyer-Olkin (KMO) tiene un valor de .918 y la prueba de esfericidad de Bartlett un grado de significación de .001. El método de extracción utilizado ha sido el análisis de componentes principales y el método de rotación Varimax con Kaiser. La rotación converge en seis iteraciones. Seis factores explican el 75,251 \% de la varianza. Dichos factores y su varianza son: Factor 1, "Satisfacción con las relaciones y el centro" 18,116 \%. Factor 2, "Satisfacción con la formación" 16,861 \%. Factor 3, "Satisfacción con los horarios" 11,539 \%, Factor 4, "Satisfacción con las instalaciones y 
recursos del centro" 9,888 \%, Factor 5, "Satisfacción con las relaciones y valoración de la inspección y administración" 9,552 \% y Factor 6, "Satisfacción con las relaciones y valoración de alumnado y familias" 9,296 \%.

Para calcular la fiabilidad de la prueba se utiliza el coeficiente de Cronbach, el cual alcanza un valor de Alpha $=.945$ (en la escala de 0 a 1) para el total de los elementos analizados.

En la tabla 1 se indican los factores obtenidos por las distintas variables analizadas así como sus pesos factoriales superiores a .30.

TABLA 1

Matriz de componentes rotados y coeficiente de Cronbach

\begin{tabular}{|c|c|c|c|c|c|c|}
\hline \multirow{2}{*}{$\begin{array}{l}\text { Variables } \\
\text { satisfacción con: }\end{array}$} & \multicolumn{6}{|c|}{ Componentes } \\
\hline & 1 & 2 & 3 & 4 & 5 & 6 \\
\hline 3. Relaciones y centro. Relación con directivos & .837 & & & & & \\
\hline $\begin{array}{l}\text { 12. Relaciones y centro. Información sobre el } \\
\text { funcionamiento del centro }\end{array}$ & .804 & & & & & \\
\hline 11. Relaciones y centro. Organización general & .797 & & & .304 & & \\
\hline $\begin{array}{l}\text { 34. Relaciones y centro. Reconocimiento por parte } \\
\text { del equipo directivo }\end{array}$ & .796 & & & & & \\
\hline 13. Relaciones y centro. Ambiente de trabajo & .767 & & & & & \\
\hline $\begin{array}{l}\text { 4. Relaciones y centro. Relación con el profesorado } \\
\text { del centro }\end{array}$ & .725 & & & & & \\
\hline $\begin{array}{l}\text { 35. Relaciones y centro. Reconocimiento por parte } \\
\text { del profesorado }\end{array}$ & .679 & & & & & \\
\hline $\begin{array}{l}\text { 30. Relaciones y centro. Participación en los órganos } \\
\text { de gobierno del centro }\end{array}$ & .653 & & .471 & & & \\
\hline 22. Formación. Gestión conflictos alumnado & & .871 & & & & \\
\hline 21. Formación. Pedagogía-psicología & & .871 & & & & \\
\hline 20. Formación. Trabajo en equipo & & .852 & & & & \\
\hline 17. Formación. Función tutorial & & .790 & & & & \\
\hline 18. Formación. Normativa educativa & & .787 & & & & \\
\hline 16. Formación. Formación permanente & & .780 & & & & \\
\hline 19. Formación. Materias que se imparten & & .763 & & & & \\
\hline 27. Horarios. Distribución horario lectivo & .357 & & .754 & & & \\
\hline 28. Horarios. Distribución horario reuniones & .455 & & .752 & & & \\
\hline
\end{tabular}




\begin{tabular}{|c|c|c|c|c|c|c|}
\hline \multirow{2}{*}{$\begin{array}{l}\text { Variables } \\
\text { satisfacción con: }\end{array}$} & \multicolumn{6}{|c|}{ Componentes } \\
\hline & 1 & 2 & 3 & 4 & 5 & 6 \\
\hline 25. Horarios. Horario laboral & & & .752 & & & \\
\hline 26. Horarios. Número de horas lectivas & & & .727 & & & \\
\hline 29. Horarios. Número de reuniones & .450 & & .711 & & & \\
\hline 8. Instalaciones y recursos. Instalaciones del centro & & & & .815 & & \\
\hline $\begin{array}{l}\text { 10. Instalaciones y recursos. Recursos materiales en } \\
\text { el centro para la enseñanza }\end{array}$ & & & & .814 & & \\
\hline $\begin{array}{l}\text { 9. Instalaciones y recursos. Recursos didácticos en el } \\
\text { centro }\end{array}$ & & & & .802 & & \\
\hline $\begin{array}{l}\text { 14. Instalaciones y recursos. Espacios para desarrollar } \\
\text { el trabajo }\end{array}$ & & & & .798 & & \\
\hline $\begin{array}{l}\text { 33. Relación y valoración con/por las instituciones. } \\
\text { Valoración por la inspección }\end{array}$ & & & & & .742 & \\
\hline $\begin{array}{l}\text { 32. Relación y valoración con/por las instituciones. } \\
\text { Valoración por la administración }\end{array}$ & & .385 & & & .722 & \\
\hline $\begin{array}{l}\text { 2. Relación y valoración con/por las instituciones. } \\
\text { Relación con la inspección }\end{array}$ & .311 & & & & .682 & \\
\hline $\begin{array}{l}\text { 38. Relación y valoración con/por las instituciones. } \\
\text { Posibilidades de promoción profesional }\end{array}$ & & & & & .662 & \\
\hline $\begin{array}{l}\text { 1. Relación y valoración con/por las instituciones. } \\
\text { Relación con la administración }\end{array}$ & .329 & & & & .635 & \\
\hline $\begin{array}{l}\text { 37. Relación y valoración con/por el alumnado y } \\
\text { familias. Valoración por las familias }\end{array}$ & & & & & & .825 \\
\hline $\begin{array}{l}\text { 6. Relación y valoración con/por el alumnado y } \\
\text { familias. Relación con el alumnado }\end{array}$ & & & & & & .809 \\
\hline $\begin{array}{l}\text { 36. Relación y valoración con/por el alumnado y } \\
\text { familias. Valoración por el alumnado }\end{array}$ & & & & & & .809 \\
\hline $\begin{array}{l}\text { 7. Relación y valoración con/por el alumnado y } \\
\text { familias. Relación con las familias }\end{array}$ & & & & & & .740 \\
\hline Coeficiente de Cronbach Total: .955 & .955 & .942 & .893 & .912 & .884 & .869 \\
\hline
\end{tabular}

Se realiza un análisis factorial confirmatorio mediante SPSS AMOS 26 con 300 sujetos. Se analiza con el método de máxima verosimilitud (ML). 


\section{FIGURA 1}

Gráfico análisis factorial confirmatorio

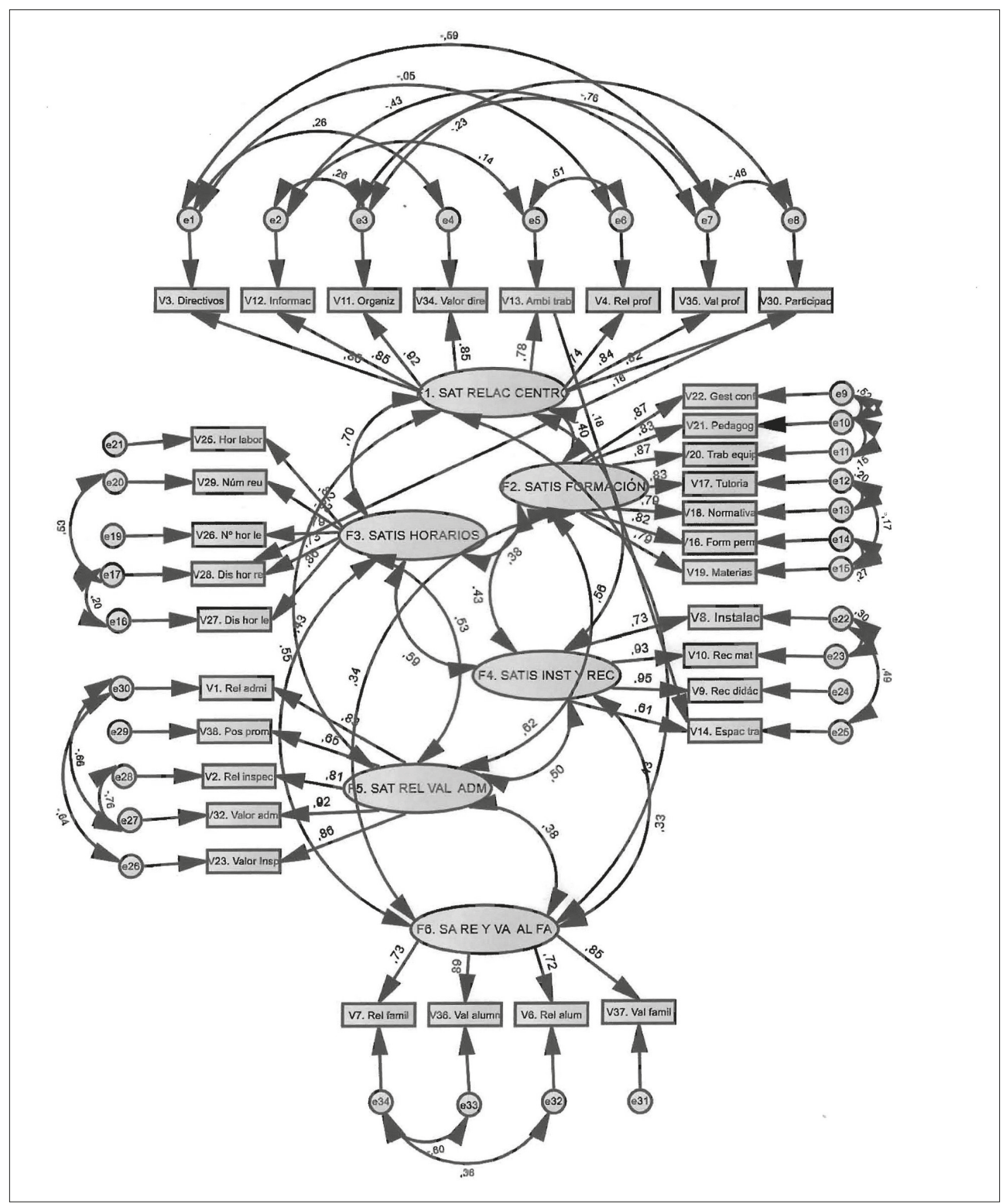

Fuente: SPSS AMOS 26.

EDETANIA 58 [Diciembre 2020], 157-182, ISSN: 0214-8560 
Los índices obtenidos son: de ajuste absoluto son chi cuadrado sobre grados libertad, PCMIN/DF = 1.979; índice de ajuste NFI = .904; índice de ajuste comparativo $\mathrm{CFI}=.949$; índice de bondad de ajuste $\mathrm{GFI}=.853$; índice de Tuker-Lewis TLI $=.941$; índice RFI $=.888$; índice normado de parsimonia PNFI $=.777$; error cuadrático medio de aproximación RMSEA $=.057$. Se concluye que el modelo cuenta con buena consistencia interna e índices de bondad de ajuste adecuados.

\section{Resultados}

3.1 Satisfacción del profesorado según género, experiencia docente, enseñanzas impartidas y tamaño del centro, en función de la titularidad

En relación con el sexo, no se encuentran diferencias respecto a la satisfacción laboral del profesorado ni en los centros públicos ni en los privadosconcertados.

Según la experiencia docente se encuentran diferencias entre el profesorado de menor experiencia, hasta 12 cursos, y el de mayor experiencia, 23 o más cursos $(7,9$ y $7,6, \rho=.024)$. Se encuentran diferencias entre dichos grupos en el caso de los centros públicos $(7,8$ y $7,6, \rho=.031)$, mientras que no existen diferencias ( $p>.050)$ en los privados-concertados.

El profesorado que imparte enseñanzas de educación infantil manifiesta mayor satisfacción en su actividad laboral que el de $\operatorname{ESO}(8,2$ y $7,5, \rho=.001)$ y el de bachillerato $(8,2$ y $7,6, \rho=.001)$. De igual manera el de educación primaria se muestra más satisfecho que el de $\operatorname{ESO}(8,0$ y $7,5, \rho=.001)$ y el de bachillerato $(8,2$ y 7,6, $\rho=.030)$. En el caso de FP, se manifiesta más satisfecho que el de $\operatorname{ESO}(8,0$ y 7,5, $\rho=.001)$ y bachillerato $(8,0$ y $7,6, \rho=.049)$. El profesorado de centros públicos que imparten enseñanzas en ESO valoran menos su satisfacción en sus tareas que el de educación infantil (7,4 y 8,2, $\rho=.001)$, el de primaria $(7,4$ y $8,0, \rho=.001)$ y el de FP $(7,4$ y $8,0, \rho=.001)$. No se encuentran diferencias ( $\mathrm{p}>.050)$ entre el profesorado de centros privados-concertados en función de las enseñanzas.

No se establecen diferencias ( $\mathrm{p}>.050$ ) en función del tamaño del centro, ni en los de titularidad pública ni en los privados-concertados. 


\subsection{Experiencia y satisfacción en cargos directivos, función tutorial, otros cargos y consejo escolar, según la titularidad del centro}

El 34,9\% de docentes manifiestan tener experiencia en cargos directivos (dirección, jefatura de estudios y/o secretaría). La media de dicha experiencia es de 7,0 cursos $(\mathrm{DT}=6,1)$. La valoración de la satisfacción es de 6,7 puntos $(\mathrm{DT}=2,2)$. El 16,1 \% le otorga menos de cinco puntos. El de los centros privados-concertados manifiesta tener mayor satisfacción en los cargos directivos que el de los centros públicos (7,8 y $6,6, \rho=.001)$.

El $90,6 \%$ de profesores y profesoras tienen experiencia en la función tutorial, con una media de 9,6 cursos, valorando la misma con 6,8 puntos. El $13,8 \%$ le otorga menos de cinco puntos. Se manifiesta más satisfecho el profesorado de los centros privados-concertados que el de los públicos $(8,2$ y $6,7, \rho=.001)$.

Dos de cada tres docentes $(66,5 \%)$ manifiestan tener experiencia en otros cargos (jefatura de departamento, coordinación de ciclo, otras jefaturas o coordinaciones), con una duración media de 6,9 cursos en ellos (DT $=6,1)$. Se valora la satisfacción con 7,0 puntos (DT $=1,9)$. El 9,7 \% le asigna menos de cinco puntos. El de los centros privados-concertados indican tener mayor satisfacción en dichas responsabilidades que el de los públicos $(7,7$ y $7,0, \rho=.001)$.

Casi la mitad de los docentes (49,8 \%) forma o ha formado parte del consejo escolar, el tiempo medio en dicha responsabilidad es de 7,9 cursos $(\mathrm{DT}=6.8)$, valorando con 6,3 puntos su satisfacción en su desempeño $(\mathrm{DT}=2,3)$. El 18,7\% le otorga menos de cinco puntos. El de centros privados-concertados manifiesta tener mayor satisfacción que el de los públicos $(6,8$ y $6,2, \rho=.016)$.

\subsection{Satisfacción del profesorado en sus relaciones interpersonales, según titularidad del centro}

El profesorado de los centros privados-concertados señala que su satisfacción respecto a sus relaciones interpersonales es significativamente mayor que el de los centros públicos en todos los aspectos estudiados, con excepción del referido a "la satisfacción con el profesorado del departamento o ciclo" en 
el que no se encuentran diferencias ( $p>.050)$. El recorrido de las valoraciones de dicha satisfacción en los centros públicos es del menor valor de 5,2 puntos al mayor de 8,0, en el caso de los privados-concertados el recorrido es de 6,4 puntos a 8,2 .

TABLA 2

Satisfacción del profesorado en sus relaciones interpersonales, según titularidad del centro

\begin{tabular}{|l|r|r|r|c|}
\hline \multirow{2}{*}{ Satisfacción del profesorado en sus relaciones con: } & \multicolumn{3}{|c|}{ Media } & \\
\cline { 2 - 5 } & Total & \multicolumn{1}{|c|}{ Públicos } & $\begin{array}{c}\text { Privados- } \\
\text { concertados }\end{array}$ & $\rho$ \\
\hline 1. La administración educativa & 5,7 & 5,5 & 6,6 & .001 \\
\hline 2. La inspección educativa & 5,2 & 5,2 & 6,4 & .001 \\
\hline 3. Los directivos del centro & 7,7 & 7,6 & 8,0 & .005 \\
\hline 4. El profesorado del centro & 7,8 & 7,7 & 8,1 & .002 \\
\hline 5. El alumnado al que se imparte docencia & 7,5 & 7,3 & 8,1 & .001 \\
\hline 6. Las familias del alumnado & 6,8 & 6,6 & 7,3 & .001 \\
\hline
\end{tabular}

3.4 Satisfacción del profesorado con el ambiente de trabajo, las instalaciones y los recursos del centro en el que desarrolla sus tareas, según titularidad del centro

El profesorado de los centros privados-concertados se manifiesta más satisfecho en su centro con el ambiente de trabajo, las instalaciones y los recursos que el de titularidad pública $(\mathrm{p}=.001)$

TABLA 3

Satisfacción del profesorado con el ambiente de trabajo, las instalaciones y recursos del centro en el que se desarrollan sus tareas, según titularidad del centro

\begin{tabular}{|l|r|r|r|r|}
\hline \multirow{2}{*}{$\begin{array}{c}\text { Satisfacción del profesorado en relación } \\
\text { con el centro respecto a: }\end{array}$} & Total & Públicos & $\begin{array}{c}\text { Privados- } \\
\text { concertados }\end{array}$ & $\rho$ \\
\cline { 2 - 5 } & 6,4 & 6,3 & 7,5 & .001 \\
\hline 1. Instalaciones & 6,6 & 6,5 & 7,6 & .001 \\
\hline 2. Recursos didácticos & & &
\end{tabular}




\begin{tabular}{|l|r|r|r|c|}
\hline \multicolumn{1}{c}{$\begin{array}{c}\text { Satisfacción del profesorado en relación } \\
\text { con el centro respecto a: }\end{array}$} & Total & Públicos & $\begin{array}{c}\text { Privados- } \\
\text { concertados }\end{array}$ & $\rho$ \\
\cline { 2 - 5 } & 6,5 & 6,4 & 7,5 & .001 \\
\hline 3. Recursos materiales & 7,1 & 7,1 & 7,7 & .001 \\
\hline 4. Organización general del centro & 7,3 & 7,3 & 7,8 & .001 \\
\hline 5. Información sobre el funcionamiento del centro & 7,5 & 7,5 & 8,0 & .001 \\
\hline 6. Ambiente de trabajo & 6,7 & 6,7 & 7,6 & .001 \\
\hline 7. Espacios de que se dispone para el trabajo & & & \\
\hline
\end{tabular}

\subsection{Satisfacción con la formación recibida, según titularidad del centro}

El profesorado de centros privados-concertados manifiesta mayor satisfacción que el de los públicos respecto a los aspectos analizados sobre su formación $(\mathrm{p}=.001)$

TABLA 4

Satisfacción con la formación recibida, según titularidad del centro

\begin{tabular}{|l|r|r|r|r|}
\hline \multirow{2}{*}{\multicolumn{1}{c|}{ Satisfacción respecto a la formación: }} & \multicolumn{4}{|c|}{ Media } \\
\cline { 2 - 6 } & Total & Públicos & $\begin{array}{c}\text { Privados- } \\
\text { concertados }\end{array}$ & \multicolumn{1}{c|}{$\rho$} \\
\hline 1. Inicial en los estudios universitarios & 5,5 & 5,4 & 6,6 & .001 \\
\hline 2. Permanente & 6,2 & 6,1 & 7,5 & .001 \\
\hline 3. Función tutorial & 4,9 & 4,8 & 6,3 & .001 \\
\hline 4. Normativa educativa & 4,6 & 4,5 & 6,0 & .001 \\
\hline 5. Materias que se imparten & 5,5 & 5,4 & 7,0 & .001 \\
\hline 6. Trabajo en equipo & 4,9 & 4,7 & 6,8 & .001 \\
\hline 7. Aspectos de pedagogía y psicología & 4,6 & 4,5 & 6,4 & .001 \\
\hline 8. Gestión de conflictos del alumnado & 4,7 & 4,6 & 6,4 & .001 \\
\hline
\end{tabular}

\subsection{Satisfacción en aspectos laborales, según titularidad del centro}

Los docentes de los centros privados-concertados manifiestan tener una mayor satisfacción en sus aspectos laborales y de reconocimiento y valora- 
ción que reciben de su trabajo que sus colegas de titularidad pública. Tan solo no se encuentran diferencias ( $\mathrm{p}>$.050) en relación con la satisfacción respecto a "las retribuciones", "la distribución del horario de reuniones" y "el número de reuniones".

TABLA 5

Satisfacción de aspectos laborales, según titularidad del centro

\begin{tabular}{|c|c|c|c|c|}
\hline \multirow[b]{2}{*}{ Satisfacción respecto a: } & \multicolumn{4}{|c|}{ Media } \\
\hline & Total & Públicos & $\begin{array}{l}\text { Privados- } \\
\text { concertados }\end{array}$ & $\rho$ \\
\hline 1. Ubicación del centro & 7,6 & 7,6 & 8,4 & .001 \\
\hline 2. Retribuciones & 6,6 & 6,4 & 6,7 & - \\
\hline 3. Horario laboral de permanencia en el centro & 7,3 & 7,3 & 7,6 & .048 \\
\hline 4. Número de horas lectivas & 6,7 & 6,7 & 7,2 & .001 \\
\hline 5. Distribución del horario lectivo & 7,2 & 7,1 & 7,7 & .001 \\
\hline 6. Distribución del horario de reuniones & 6,9 & 6,9 & 7,2 & - \\
\hline 7. Número de reuniones & 6,8 & 6,8 & 7,1 & - \\
\hline $\begin{array}{l}\text { 8. Posibilidades de participación en los órganos de } \\
\text { gobierno del centro }\end{array}$ & 7,0 & 7,0 & 7,4 & .014 \\
\hline 9. Distancia desde el domicilio al centro & 7,5 & 7,4 & 8,3 & .001 \\
\hline \multicolumn{5}{|c|}{ 10. Reconocimiento y valoración que se realiza del trabajo del profesorado por parte de: } \\
\hline 10. 1. Las administraciones educativas & 4,1 & 3,9 & 5,5 & .001 \\
\hline 10. 2. La inspección educativa & 4,3 & 4,2 & 5,6 & .001 \\
\hline 10. 3. El equipo directivo del centro & 6,9 & 6,9 & 7,6 & .001 \\
\hline 10. 4. El profesorado del centro & 6,9 & 6,9 & 7,4 & .001 \\
\hline 10. 5. El alumnado al que se imparte docencia & 7,1 & 7,1 & 7,9 & .001 \\
\hline 10. 6. Las familias del alumnado & 6,5 & 6,4 & 7,3 & .001 \\
\hline 11. Posibilidades de promoción profesional & 4,8 & 4,6 & 6,2 & .001 \\
\hline 12. Trabajo que se realiza como docente & 7,8 & 7,7 & 8,3 & .001 \\
\hline 13. Situación respecto a hace cinco cursos & 2,7 & 2,7 & 2,4 & .001 \\
\hline
\end{tabular}




\subsection{Posibles situaciones de acoso, maltrato o agresión al profesorado, según titularidad del centro}

En relación con las posibles situaciones de "acoso, maltrato o agresión" sufridas por el profesorado, se valora las recibidas por parte de "administraciones educativas", "inspección educativa", “equipos directivos”, "profesorado", "alumnado" y "familias del alumnado". Para las respuestas se proponían dos opciones, "sí" y "no". El 27,5 \% del profesorado manifiesta sufrir situaciones de "acoso, maltrato o agresión" por parte del "alumnado", y el 23,8 \% por "algún familiar del alumnado". Los "miembros de los equipos directivos" son los responsables del 19,4\% de las situaciones sufridas. El 17,7 \% considera que sufre o ha sufrido dichas situaciones por parte de "algún profesor o profesora". Los "responsables de las administraciones educativas" son señalados por el 12,2\%. Por parte de "la inspección educativa" es el 7,9 \% el que se siente "acosado, maltratado o agredido".

El profesorado de los centros públicos manifiestan mayor porcentaje de "acoso, maltrato o agresión" que los de los privados-concertados, con excepción de lo relativo a por "algún familiar del alumnado" en el que los porcentajes son similares.

TABLA 6

Posibles situaciones de acoso, maltrato o agresión, según titularidad del centro

\begin{tabular}{|l|r|r|r|}
\hline \multirow{2}{*}{$\begin{array}{c}\text { Posibles situaciones de acoso, maltrato o agresión sufridas } \\
\text { por parte de: }\end{array}$} & \multicolumn{3}{c|}{ Si \% } \\
\cline { 3 - 5 } & Total & Públicos & $\begin{array}{c}\text { Privados- } \\
\text { concertados }\end{array}$ \\
\hline 1. Responsable de las administraciones educativas & 12,2 & 14,7 & 5,0 \\
\hline 2. Miembros del servicio de inspección educativa & 7,9 & 8,3 & 2,3 \\
\hline 3. Miembros de equipos directivos & 19,4 & 20,2 & 11,4 \\
\hline 4. Profesorado & 17,7 & 17,9 & 14,4 \\
\hline 5. Alumnado & 27,5 & 28,8 & 12,3 \\
\hline 6. Familia del alumnado & 23,8 & 23,9 & 23,7 \\
\hline
\end{tabular}


3.7.1 Posibles situaciones de acoso, maltrato o agresión sufridas por el profesorado según titularidad del centro y su gravedad

El profesorado que ha manifestado sufrir las situaciones señaladas ha valorado en una escala su gravedad: "muy grave", "bastante grave", "algo grave" o "leve". Se consideran "muy graves" o "bastante graves", las sufridas por parte del "alumnado" en el 9,5\% de los casos (el 10,0 \% centros públicos y $3,5 \%$ privados-concertados), por las "familias del alumnado" el 8,1 \% (el $8,1 \%$ y $8,3 \%$ ), por "equipos directivos" el 8,1\% (8,6 \% y 2,6 \%), por "profesorado" el 6,6\% (6,9\% y 3,5\%), por "las administraciones educativas" el $5,0 \%(5,9 \%$ y $3,9 \%)$ y por "el servicio de inspección educativa" el 3,8 \% $(3,9 \%$ y $2,6 \%)$.

TABLA 7

Posibles situaciones de acoso, maltrato o agresión sufridas por el profesorado según su gravedad, en función de la titularidad del centro

\begin{tabular}{|l|r|r|r|r|r|}
\hline \multirow{2}{*}{$\begin{array}{c}\text { Posibles situaciones de acoso, maltrato } \\
\text { o agresión sufridas por parte de: }\end{array}$} & \multirow{2}{*}{ Total } & \multicolumn{4}{|c|}{ Grado de acoso, maltrato o agresión } \\
\cline { 3 - 6 } & & $\begin{array}{c}\text { 1. Muy } \\
\text { grave }\end{array}$ & $\begin{array}{c}\text { 2. Bastante } \\
\text { grave }\end{array}$ & $\begin{array}{c}\text { 3. Algo } \\
\text { grave }\end{array}$ & 4. Leve \\
\hline $\begin{array}{l}\text { 1. Responsables de las administraciones } \\
\text { educativas }\end{array}$ & 12,2 & 1,5 & 3,5 & 3,1 & 4,1 \\
\hline 2. Miembros inspección educativa & 7,9 & 1,5 & 2,3 & 2,0 & 2,1 \\
\hline 3. Miembros de equipo directivo & 19,4 & 2,7 & 5,4 & 5,8 & 5,5 \\
\hline 4. Profesor o profesora & 17,7 & 2,2 & 4,4 & 5,1 & 6,0 \\
\hline 5. Alumno o alumna & 27,5 & 3,1 & 6,4 & 8,4 & 9,6 \\
\hline 6. Familia del alumnado & 23,8 & 2,5 & 5,6 & 6,9 & 8,8 \\
\hline
\end{tabular}

\section{DisCusión y CONCLUSIONES}

La satisfacción laboral del profesorado se relaciona con la calidad de la enseñanza en diversos trabajos (Anaya-Nieto y López-Martín, 2014; Banerjee et al., 2017; Demirtaú, 2010; Gil-Flores, 2017; Persevica, 2011). Se ha procedido a analizar la titularidad del centro como factor que puede influir en dicha 
satisfacción y por tanto en la posible mejora de la calidad educativa. En los trabajos sobre la satisfacción laboral del profesorado se analizan muchos de sus aspectos, no obstante, el de la titularidad de los centros en los que ejerce sus funciones el profesorado no es tratado con frecuencia.

Respecto al análisis factorial confirmatorio realizado se concluye que el modelo cuenta con buena consistencia interna e índices de bondad de ajuste adecuados.

El nivel de satisfacción laboral del profesorado en el presente trabajo se valora como medio-alto, semejante al señalado por Anaya y Suárez (2007) y Larkin et al., (2016) y en la línea de lo aparecido en el informe Pisa (2019). Se constata en este trabajo que, en general, el profesorado de los centros privados-concertados se manifiesta más satisfecho laboralmente que el de los públicos tal como señalan, asimismo, distintos autores (Crossman \& Harris, 2006; Gius, 2015; Naeem-Akhtara et al., 2010; Sungu et al., 2014).

En el presente estudio no se encuentran diferencias sobre la satisfacción del profesorado desde hace cinco cursos al momento actual, mientras que, por el contrario, en los estudios longitudinales se constata que la satisfacción laboral del profesorado disminuye con el tiempo (Anaya-Nieto y López-Martín, 2014; Gil-Flores, 2017; Klassen \& Anderson, 2009; MetLife Foundation, 2012).

En relación con el sexo y la satisfacción laboral del profesorado, no existe acuerdo en las investigaciones analizadas respecto a la existencia de diferencias. En este trabajo no se encuentran diferencias, coincidiendo con lo señalado en algunos estudios (Crossman y Harris, 2006; Muñoz-Menéndez et al., 2017; Skaalvik y Skaalvik, 2009).

En relación con el tiempo de experiencia docente y la satisfacción laboral del profesorado tampoco existe unanimidad en las investigaciones. En distintos trabajos se observa que con el aumento de la experiencia docente disminuye la satisfacción laboral (Anaya-Nieto y Suárez, 2006; Anaya-Nieto y López-Martín, 2014; Gil-Flores, 2017; Skaalvic y Skaalvic, 2009; Xin y MacMillan, 1999). En el presente trabajo el grupo de menor experiencia docente de los centros públicos manifiesta mayor satisfacción que el de mayor experiencia, no encontrándose diferencias al respecto en el de los privadosconcertados. 
En los análisis realizados se constata que el profesorado de educación infantil y el de educación primaria manifiestan una mayor satisfacción que el de ESO y bachillerato, conclusiones que coinciden con las de diversos trabajos (Anaya-Nieto y López-Martín, 2014; Cantón-Mayo y Téllez-Martínez, 2016; Martín-Ramos 2015; Muñoz-Menéndez et al., 2017).

Para Papanastasiou y Zembylas (2005) el profesorado que ocupa puestos de mayor responsabilidad en las instituciones educativas tiende a estar más satisfecho con su trabajo, tal como se concluye en este análisis. La satisfacción respecto a la dirección del centro, función tutorial, otros cargos y como miembros del consejo escolar es mayor en el caso de los centros privadosconcertados que en los públicos.

La satisfacción del profesorado en sus relaciones con la administración educativa no parece ser muy adecuada (Hernández-Silva et al., 2017; Torres, 2010). En este trabajo se otorga una satisfacción "media" al respecto siendo menos valorada por el de los centros públicos. Distintos estudios señalan que el profesorado se siente satisfecho en sus relaciones con el alumnado (Hernández-Silva et al., 2017; Torres, 2010; Veldman et al., 2013) tal como se indica, asimismo, en el análisis que se presenta, tanto en el caso del profesorado de los centros públicos como en el de los privados-concertados.

Las condiciones del lugar de trabajo son bien valoradas por el profesorado consultado, coincidiendo con lo señalado en el National Center For Education Statistics (1997) y Santos-Rego et al. (2010). En todos los aspectos estudiados en este trabajo se observa una mayor satisfacción por parte del profesorado de los centros privados-concertados. Respecto a la retribución se entiende en algunas investigaciones que tiene un efecto positivo sobre la satisfacción laboral (Franco-López et al., 2020; Persevica, 2011), así se observa en este estudio, no estableciéndose diferencias según la titularidad del centro.

Ante la grave cuestión de sufrir o haber sufrido situaciones de "acoso, maltrato o agresión", un importante porcentaje así lo señala, fundamentalmente respecto al "alumnado" y a sus "familias", y aunque en menor medida, pero de manera también importante, por parte del "equipo directivo" y "profesorado". En algunos estudios se señala que el estrés y la ansiedad disminuyen la satisfacción laboral (Klassen y Chiu 2010; Liu y Ramsey, 2008). 
En general, podemos señalar que en el presente análisis se observa que respecto a la satisfacción laboral del profesorado en relación con el género no se encuentran diferencias en función de la titularidad del centro. El grupo de menor experiencia docente en centros públicos se manifiesta más satisfecho que el de mayor experiencia. El profesorado de educación infantil y el de educación primaria se manifiestan más satisfechos que el de ESO y bachillerato en el caso de los centros públicos, en los privados no se encuentran diferencias. Tampoco se encuentran diferencias según el tamaño del centro.

El profesorado de los centros privados-concertados manifiesta tener mayor satisfacción que en los centros públicos en los cargos directivos, función tutorial, en otros cargos, como miembros del consejo escolar, en sus relaciones interpersonales, con el ambiente de trabajo, las instalaciones y recursos del centro, su formación y aspectos laborales.

Es demasiado elevado el porcentaje de profesorado que manifiesta sufrir situaciones de "acoso, maltrato o agresión" por parte de las instituciones y miembros de la comunidad educativa. El profesorado de los centros públicos lo señalan en mayor porcentaje que el de los privados-concertados.

Aunque la muestra analizada es amplia y correspondiente a las diferentes comunidades autónomas de España, se considera que la obtenida del profesorado de centros privados-concertados podría mejorarse ya que es muy inferior a la de los públicos.

Cabe profundizar en el importante tema de la satisfacción laboral del profesorado. Las administraciones educativas deben tener en cuenta este aspecto en sus planificaciones para una mejora en la calidad laboral del profesorado y de la enseñanza. Asimismo, también deben considerar la baja valoración que el profesorado otorga al reconocimiento y apoyo prestado por su parte.

Las importantes diferencias que aparecen en relación con la titularidad del centro podrían analizarse y conocer las causas por las que se establecen.

Se entiende que se debe intervenir con urgencia en los graves problemas de "acoso, maltrato o agresión" que un elevado porcentaje de profesionales de la educación manifiestan sufrir. Intervención por parte de las administraciones, de los propios centros y miembros de la comunidad educativa en general e impulsar una clara concienciación social al respecto. 


\section{BiBLIOGRAFÍA}

Anaya-Nieto, D. y López-Martín, E. (2014). Satisfacción laboral del profesorado en 2012-13 y comparación con los resultados de 2003-04. Un estudio de ámbito nacional. Revista de Educación 365, 96-121. Recuperado de: https://dialnet.unirioja.es/servlet/autor?codigo $=8911$.

Anaya-Nieto, D. y SuÁRez, J. M. (2006). La satisfacción laboral de los profesores en función de la etapa educativa, del género y de la antigüedad profesional. Revista de Investigación Educativa 24(2), 541-556. Recuperado de: https://revistas.um.es/rie/article/view/97181.

Anaya-Nieto, D. y SuÁrez, J. M. (2007). Satisfacción laboral de los profesores de educación infantil, primaria y secundaria: Un estudio de ámbito nacional. Revista de Educación 344, 217-243. Recuperado de: https://n9.cl/ cmfj.

Banerjee, N., Stearns, E., Moller, S. y Mickelson, R. A. (2017). Teacher job satisfaction and student achievement: The roles of teacher professional community and teacher collaboration in schools. American Journal of Education 123(2), 1-39. Recuperado de: https://doi.org/10.1086/689932.

Cantón-Mayo, I. y Téllez-Martínez, S. (2016). La satisfacción laboral y profesional de los profesores. Revista Lasallista de Investigación 13(1), 214-226. Recuperado de: https://www.redalyc.org/pdf/695/69545978019. pdf.

Collie, R. J., Shapka, J. D. y Perry, N. E. (2012). School climate and social-emotional learning: Predicting teacher stress, job satisfaction and teaching efficacy. Journal of Educational Psychology 104(4), 1189-1204. Recuperado de: https://doi.org/10.1037/a0029356.

Crossman, A. y Harris, P. (2006). Job satisfaction of secondary school teachers. Educational Management Administration and Leadership 34, 29-46. Recuperado de: https://journals.sagepub.com/doi/10.1177/1741143206059538.

De Frutos, J. A., González, P., Maíllo, A., Peña, J. I. y Riesco, M. (2007). Condiciones de trabajo y satisfacción laboral de los docentes en las escuelas católicas de Madrid. Educación y Futuro 17, 9-42. Recuperado de: https://dialnet.unirioja.es/servlet/articulo? codigo $=2392447$. 
Demirtaú, Z. (2010). Teachers' job satisfaction levels. Procedia Social and Behavioral Sciences 9, 1069-1073. Recuperado de: https://doi.org/10.1016/ j.sbspro.2010.12.287

Ferguson, K., Frost, L. y Hall, D. (2012). Predicting teacher anxiety, depression, and job satisfaction. Journal of Teaching and Learning 8(1), 2742. Recuperado de: https://n9.cl/muo0b.

Franco-López, J.A., López-Arellano, H. y Arango-Botero, D. (2020). La satisfacción de ser docente: un estudio de tipo correlacional. Revista Complutense de Educación 31(1), 55-67. Recuperado de: https://dialnet.unirioja . es/servlet/articulo? codigo $=7214111$.

GiL-Flores, J. (2017). Características personales y de los centros educativos en la explicación de la satisfacción laboral del profesorado. Revista de Psicodidáctica 22(1). Recuperado de: https://bit.ly/331XDPu.

GiUs, M. (2015). A comparison of teacher job satisfaction in public and private schools. Academy of Educational Leadership Journal 19(3), 155-164. Recuperado de: https://www.questia.com/library/journal/1P3-3966658041/ a-comparison-of-teacher-job-satisfaction-in-public.

GüELL, L. (2014): Estudio de la satisfacción laboral de los maestros (tesis doctoral). Universitat Internacional de Catalunya. Recuperado de: https://www. tdx.cat/bitstream/handle/10803/293783/Tesi_Luisa_G\%C3\%BCell_ Malet.pdf?sequence $=1$.

Hanushek, E. A., Kain, J. F. y Rivkin, S. G. (2004). Why public schools lose teachers. Journal of Human Resources 39, 326-354. Recuperado de: http:// jhr.uwpress.org/content/XXXIX/2/326.abstract.

Hernández-Silva, C., Pavez-Lizarraga, A., González-Donoso, A. y TecPanFlores, S. (2017). ¿Se sienten valorados los profesores en Chile? Educación y educadores 20(3), 434-447. Recuperado de: https:/educacionyeducadores.unisabana.edu.co/index.php/eye/article/view/7376.

Hernando-Mora, I. y SAnz-Ponce, R. (2015). La percepción del profesorado de educación secundaria ante la conflictividad escolar. EDETANIA. Estudios y propuestas socio-educativas 48, 41-60. Recuperado de: https:// dialnet.unirioja.es/servlet/articulo?codigo $=5349084$.

Klassen, R. M. y Anderson, C. J. K. (2009). How times change: Secondary teachers' job satisfaction and dissatisfaction in 1962 and 2007. British 
Educational Research Journal 35(5), 745-759. Recuperado de: https://onlinelibrary.wiley.com/doi/abs/10.1080/01411920802688721.

Klassen, R. M. y Chiu, M. M. (2010). Effects on teachers' self-efficacy and job satisfaction: Teacher gender, years of experience, and job stress. Journal of educational Psychology 102(3), 741-756. Recuperado de: https:// doi.org/10.1037/a0019237.

Larkin, I. M., Brantley-Dias, L. y LoKey-VegA, A. (2016). Job satisfaction, organizational commitment, and turnover intention of online teachers in the K-12 setting. Online Learning 20(3), 26-51. Recuperado de: https:// eric.ed.gov/?id=EJ1113339.

Liu, X. S. y Ramsey, J. (2008). Teachers' job satisfaction: Analyses of the teacher follow-up survey in the United States for 2000-2001. Teaching and Teacher Education 24(5), 1173-1184. Recuperado de: https://doi. org/10.1016/j.tate.2006.11.010.

MARTín-RAmos, A. (2015). La satisfacción laboral y su relación con el clima organizacional en el ámbito educativo. Universidad de La Laguna: Trabajo Final de Grado. Recuperado de: https://riull.ull.es/xmlui/handle/915/1094. Martínez-Garrido, C. (2017). La incidencia del liderazgo y el clima escolar en la satisfacción laboral de los docentes en América Latina. Archivos de análisis de politicas de educación 25(80), 1-22. Recuperado de: https:// epaa.asu.edu/ojs/article/view/2851/1943.

MetLife Foundation. (2012). The MetLife Survey of the American Teacher: Teachers, parents, and the economy. MetLife, Inc. Recuperado de: http:// files.eric.ed.gov/fulltext/ED530021.pdf.

Ministerio de Educación y Formación Profesional. Instituto Nacional de Evaluación Educativa (2019). Informe PISA 2018. Recuperado de: https:// www.educacionyfp.gob.es/inee/evaluaciones-internacionales/pisa/pisa2018.html.

MitRA, S. (2018). Job satisfaction: A comparative study among government and private school teacher's. International Journal of Academic Research and Development 3(2), 583-585. Recuperado de: http://www.academicjournal.in/archives/2018/vol3/issue2.

Muñoz-MÉndez, T., Gómez-Mármol, A. y SÁnchez-Alcaraz, B. (2017). Satisfacción laboral en los docentes de educación infantil, primaria y secun- 
daria. Revista Gestión de la Educación 7(1), 161-177. Recuperado de: https://revistas.ucr.ac.cr/index.php/gestedu/article/view/27578.

Naeem-Akhtara, S., Amir-Hashmib, M. y Hussain-Naqvic, S. I. (2010). A comparative study of job satisfaction in public and private school teachers at secondary level. Procedia Social and Behavioral Sciences 2, 42224228. Recuperado de: https://www.sciencedirect.com/science/article/pii/ S1877042810007081.

National Center for Education Statistics (1997). Job satisfaction among America's teachers: effects of workplace conditions, background characteristics, and teacher compensation. Washington, D.C., Office of Educational Research and Improvement, US Department of Education. Recuperado de: https://nces.ed.gov/pubs97/97471.pdf.

Nigama, K., Selvabaskar, S., Surulivel, T., Alamelu, R. y Uthaya (2018). Job satisfaction school among school teacher. International Journal of Pure and Applied Mathematics 119(7), 2645-2655. Recuperado de: https:// acadpubl.eu/jsi/2018-119-7/articles/7c/80.pdf.

Papanastasiou, E. C. y Zembylas, M. (2005). Job satisfaction variance among public and private kindergarten school teachers in Cyprus. International Journal of Educational Research 43(3), 147-167. Recuperado de: https:// doi.org/10.1016/j.ijer.2006.06.009.

Persevica, A. (2011). The significance of the teacher's job satisfaction in the process of assuring quality education. Problems of education in the 21century 34, 98-109. Recuperado de: http://www.scientiasocialis.lt/pec/files/ pdf/vol34/98-109.Persevica_Vol.34.pdf.

RAMERY-GelPI, E. (2017). La satisfacción laboral del director escolar y los docentes en los centros de secundaria de Orlando, Florida (tesis doctoral). Universidad de Jaén. Recuperado de: https://dialnet.unirioja.es/servlet/ tesis? codigo $=139853$.

Raso-Sánchez, F., Sola-Martínez, T. y Hinojo-Lucena, F. J. (2017). Satisfacción del profesorado de la escuela rural de la provincia de Granada (España) respecto a la organización escolar. Bordón 69 (2), 79-96. Recuperado de: https://dialnet.unirioja.es/servlet/autor?codigo $=499125$.

Ros-Garrido, A. y GarcíA-Rubio, J. (2016). La calidad en la formación del profesorado del sistema educativo y de los certificados de profesionalidad. 
EDETANIA. Estudios y propuestas socio-educativas 50, 101-119. Recuperado de: https://dialnet.unirioja.es/servlet/articulo? codigo=6039916.

Santos-Rego, M. A., Godás-Otero, A., Lorenzo-Moledo, M. M. y Gómez-Fraguela, J. A. (2010). Eficacia y satisfacción laboral de los profesores no universitarios: Revisión de un instrumento de medida. Revista Española de Pedagogía 68(245), 151-170. Recuperado de: https://dialnet. unirioja.es/servlet/articulo?codigo $=3099491$.

Schwartz, G. J. (2017). The relationship between teacher job satisfaction and principal leadership styles (tesis doctoral). Jefferson City, Tennessee. USA: Carson-Newman University. Recuperado de: https://www.cn.edu/ libraries/tiny_mce/tiny_mce/plugins/filemanager/files/Dissertations/DissertaionsFall2017/Gwendolin_J._Schwartz.pdf.

SkAALVIK, E. M. y SkAALVIK, S. (2009). Does school context matter? Relations with teacher burnout and job satisfaction. Teaching and Teacher Education 25(3), 518-524. Recuperado de: https://doi.org/10.1016/j.tate.2008.12.006. Sotomayor-Atahuamán, L. (2020). Acompañamiento pedagógico y satisfacción laboral en instituciones educativas públicas de la Red $N^{\circ} 16$ de Villa El Salvador (trabajo de fin de grado). Universidad César Vallejo. Recuperado de: http://repositorio.ucv.edu.pe.

Sungu, H., Ilgan, A., Parylo, O. y Erdem, M. (2014). Examining teacher job satisfaction and principals' instructional supervision behaviors: A comparative study of Turkish private and public school teachers. Alberta Journal of Educational Research 60(1), 98-118. Recuperado de: https://eric.ed.gov /?id=EJ1048971.

Torres, J. A. (2010). Análisis del grado de satisfacción del profesorado de educación secundaria en el desarrollo de su labor docente. Contextos Educativos 13, 27-41. Recuperado de: https://publicaciones.unirioja.es/ojs/index.php/contextos/article/view/624/587.

Veldman, I., Van Tartwijk, J., Brekelmas, M. y Wubbles, T. (2013). Job Satisfaction and teacher-student relationships across the teaching career: Four case studies. Teaching and Teacher Education 32, 55-65. Recuperado de: https://eric.ed.gov/?id=EJ1003342.

XIN, M. y MacMillan, R. B. (1999) Influences of Workplace Conditions on Teachers' Job Satisfaction. The Journal of Educational Research 93(1), 3947. Recuperado de: https://eric.ed.gov/?id=EJ598250. 